\title{
Lack of miR-216b-5p, miR-33a-5p or miR-122-5p in MSCs exosomes promotes breast cancer glycolysis by targeting HK2, LDHA and PKM2
}

\author{
Jiyi Xia ${ }^{1, *}$, Xiaolan Yư ${ }^{2, *}$, Xiguang $\mathrm{Mao}^{3}$, Hongyuan An ${ }^{4}$, Xiaoping Tang ${ }^{5}$, Xiaoyan \\ Wang $^{5}$, Tao He ${ }^{1}$ and Yong Liu ${ }^{6}$ \\ ${ }^{1}$ Cancer Research Institute of SouthWest Medical University, Luzhou, Sichuan, China \\ ${ }^{2}$ Department of Obstetrics and Gynecology, Affiliated TCM Hospital of SouthWest Medical University, Luzhou, Sichuan, China \\ ${ }^{3}$ Department of Obstetrics and Gynecology, Affiliated Hospital of SouthWest Medical University, Luzhou, Sichuan, China \\ ${ }^{4}$ The Second Ward of Surgery (Breast Surgery), Luzhou People's Hospital, Luzhou, Sichuan, China \\ ${ }^{5}$ Experimental Medicine Center, The Affiliated Hospital of SouthWest Medical University, Luzhou, Sichuan, China \\ ${ }^{6}$ Department of Pathology, Affiliated Hospital of Southwest Medical University, Luzhou, Sichuan, China \\ *These authors have contributed equally to this work \\ Correspondence to: Tao He, email: hetaipeace@163.com \\ Yong Liv, email: liuyong_lz@126.com
}

Keywords: MSC; exosomes; breast cancer; miRNA

Received: April 27, $2017 \quad$ Accepted: October 28, $2017 \quad$ Published: January 02, 2018

Copyright: Xia et al. This is an open-access article distributed under the terms of the Creative Commons Attribution License 3.0 (CC BY 3.0), which permits unrestricted use, distribution, and reproduction in any medium, provided the original author and source are credited.

\section{ABSTRACT}

Exosomes contain many important components including miRNAs for cancerstromal communication. Mesenchymal stromal cells support tumor development, however, the low expression of miRNAs in exosomes and their regulation roles from mesenchymal stromal cells are still unclear. In this study, we hypothesized that mesenchymal stromal cells-derived exosomes promote breast cancer cell glycolysis. Our results showed that mesenchymal stromal cells-derived exosomes promoted cell proliferation and glycolysis of breast cancer cells. Furthermore, sequencing of exosomal RNAs revealed miR-216b-5p, miR-33a-5p and miR-122-5p were lack of expression in exosomes from mesenchymal stromal cells. MiR-216b-5p, miR-33a-5p and miR-122-5p overexpression in breast cancer cells suppressed glycolysis by targeting HK2, LDHA and PKM2 respectively. These results provided valuable insights and mechanism of mesenchymal stromal cells-secreted exosomes on the glycolysis of breast cancer.

\section{INTRODUCTION}

Mesenchymal stromal cells (MSCs) are important cells of tumor stroma. MSCs potentially support tumor development through immune suppression, epithelial mesenchymal transition, angiogenesis, and serve as cancer stromal cells [1-3]. It is verified that bone marrow MSCs is the main source of carcinoma associated fibroblasts in breast cancer microenvironment [3]. Breast cancer is the most common cancer from women and the role of tumor microenvironment in breast cancer progression is studied widely, but the relationship between MSCs and breast cancer cells is still unclear [4].
Exosomes are small membrane-bound nanovesicles with the characteristic size of $30-120 \mathrm{~nm}$ in diameter [5]. Exosomes are generated from endosomal origins constitutively, released by various cells, and more frequently by tumor cells [6-8]. Exosomes are actively secreted from cells by an exocytosis pathway used for receptor removal and crosstalk between cells. Exosomes contain many important components such as proteins, RNA, DNA, and lipids for cancer-stromal communication [8-9]. Specifically, cell-secreted microRNAs (miRNAs) with the length of 18-22 nucleotides are predominantly carried by exosomes [10]. MiRNAs have been studied in recent years for their roles in post-transcriptional 
regulation of gene expression through mRNA silencing [10]. Therefore, understanding the functions of the MSCs derived exosomes in cancer is critical to elucidate the cross-talk between MSCs and breast cancer cell biological functions including glycolysis.

Glycolysis is a most common characteristic in various cancers. The enzymes directly regulating glycolysis have also been implicated in promoting a drugresistant phenotype. There are many factors for promoting glycolysis of cancer such as hypoxia, acidic state, tumor stroma cell interaction and others. In the progression of cancer glycolysis, hexokinase-2 (HK2) is the first ratelimiting enzyme in the glycolytic pathway and is upregulated in many cancers. PKM2 is the final rate-limiting enzyme of the glycolytic pathway and is involved in the conversion of phosphoenolpyruvate and ADP into pyruvate and ATP. PKM2 is highly expressed in cancers. Lactate dehydrogenase A (LDHA) catalyzes the conversion of pyruvate to lactate and is considered to be a key checkpoint of anaerobic glycolysis. It is elevated in many types of cancers and has been linked to tumor growth, maintenance, and invasion; therefore, its inhibition may restrict the energy supply in tumors and thereby reduce the metastatic and invasive potential of cancer cells.

However, the molecular mechanism for breast cancer glycolysis is far from elucidated. In this study, we hypothesized that human MSCs-secreted exosomes have important on the regulation of breast cancer cell glucose metabolism. Hence, we investigated the effect and molecular mechanism of MSCs-secreted exosomes on breast cancer cell glycolysis.

\section{RESULTS}

\section{MSCs -derived exosomes promotes breast cancer cell proliferation}

Exosomes were isolated from serum-free culture medium of MSCs through a series of centrifugation and filtration steps. The markers of exosomes including CD63 and CD81 were detectable (Figure 1A-1B). Cell growth was evaluated in MCF-7, MDA-MB-468 and MDAMB-231 cells treated with MSCs-exosomes. The data showed that MSCs-exosomes increased cell proliferation of breast cancer cells than the controls (Figure 1C-1E). These data demonstrated that MSCs-exosomes promoted breast cancer cell growth.

\section{MSCs-derived exosomes promotes breast cancer cell glycolysis}

In order to evaluate the impact of MSCs-exosomes on glycolysis, we measured the lactic acid production via extracellular acidification rate (ECAR). Culturing breast cancer cells with MSCs-exosomes led to a marked increase in ECAR (Figure 2A-2C). ATP production in the breast cancer cells with exosomes treatment was enhanced (Figure 2D-2F). Oxygen consumption rate (OCR) indicates for mitochondrial respiration. OCR was measured in breast cancer cells treated with or without exosomes and OCR decreased (Figure 2G-2I). Consequently, the expression levels of key enzymes controlling glycolysis HK2, LDHA, PDK-1 and PKM2 were significantly higher in those cells including (Figure 2G-2I). The data indicated that breast cancer cells became more glycolytic in the present of exosomes (Figure 2J).

\section{MSCs-derived exosomes carrying some significant miRNAs}

Exosomes contain proteins, mRNA, miRNAs and others depending on the cell type and their environment. To further explore the mechanism of MSCs-derived exosomes promoting breast cancer cell proliferation and glycolysis, exosomes were performed for miRNA array analysis. The miRNA array was shown in Figure $3 \mathrm{~A}$. Some of low levels of miRNAs from the array were selected for real time RT-PCR analysis and it was found that miR-216b-5p, miR-33a-5p and miR-122-5p were lack of expression in the exosomes from MSCs (Figure 3B3D).

\section{MiR-216b-5p, miR-33a-5p and miR-122-5p contribute to exosomes-mediated glucose metabolism}

In order to evaluate the impact of miR-216b-5p, miR-33a-5p and miR-122-5p on glucose metabolism of breast cancer cells, we measured ECAR. MCF-7 and MDA-MD-231 cells were transfected with miR-216b-5p, miR-33a-5p and miR-122-5p led to a marked decrease in ECAR under baseline conditions and upon glucose addition (Figure 4A-4B). MiR-216b-5p, miR-33a-5p and miR-122-5p resulted in a significantly decreased ATP production (Figure 4C-4D) and increased OCR (Figure 4E-4F).

\section{Target glucose associated genes of miR-216b-5p, miR-33a-5p and miR-122-5p in breast cancer cells}

To find the target genes of miR-216b-5p, miR$33 a-5 p$ and miR-122-5p which are associated with glucose metabolism, miRBase and Targetscan7.1 tools were used to predict the target genes. We found that HK2 was a target gene of miR-216b-5p; LDHA was a target gene of miR-33a-5p and PKM2 was a target gene of miR-122-5p (Figure 5A). To know whether miR-216b-5p, miR-33a-5p and miR-122-5p directly binds the 3 'UTR region of $\mathrm{HK} 2$, LDHA and PKM2, we generated some chimeric constructs which harbor luciferase wild-type 3'UTR sequence (WT$3^{\prime}$ UTR) or xmutant 3'UTR sequence (Mut-3'UTR). As 
expected, miR-216b-5p, miR-33a-5p and miR-122-5p mimics exclusively inhibited the luciferase activity of Luci-WT-3'UTR, suggesting that the putative binding site is important for miR-216b-5p, miR-33a-5p and miR122-5p suppressing HK2, PKM2 and LDHA expression (Figure 5B-5D).

\section{MiR-216b-5p, miR-33a-5p and miR-122-5p regulate HK2, LDHA and PKM2 expression in breast cancer cells}

From Figure 5, we knew that miR-216b-5p, miR$33 a-5 p$ and miR-122-5p may regulate HK2, LDHA and PKM2 expression in MCF-7 and MDA-MB-231 cells. To further confirm whether miR-216b-5p, miR-33a-5p and miR-122-5p regulate HK2, LDHA and PKM2 expression on post-transcriptional levels, MCF-7 and MDA-MB-231 cells were transfected with miR-216b-5p, miR-33a-5p and miR-122-5p mimics and miRNA controls, HK2, LDHA and PKM 2 mRNA and protein were evaluated by real time RT-PCR and western blotting respectively. The data showed that HK2, LDHA and PKM2 mRNAs were down-regulated MCF-7 and MDA-MB-231 cells (Figure 6A-6C). The protein levels of HK2, LDHA and PKM2 from the cells with miR-216b-5p, miR-33a-5p and miR122-5p transfection decreased (Figure 6D-6E). To confirm that miR-216b-5p, miR-33a-5p and miR-122-5p may regulate HK2, LDHA and PKM2 expression, MCF-7 and MDA-MB-231 cells were transfected with miR-216b-5p, miR-33a-5p and miR-122-5p inhibitors or their controls to detect the target genes' expression. We found that HK2, LDHA and PKM2 mRNA increased in MCF-7 and MDAMB-231 cells (Figure 6F-6H), so did HK2, LDHA and PKM2 protein levels (Figure 6I-6J). So, the data clearly indicated that miR-216b-5p, miR-33a-5p and miR-122-5p regulated HK2, LDHA and PKM2 expression on mRNA and protein levels in breast cancer cells.

\section{MiR-216b-5p, miR-33a-5p and miR-122-5p suppresses glycolysis by targeting HK2, LDHA and PKM2 in breast cancer cells}

In order to evaluate the impact of miR-216b-5p, miR$33 a-5 p$ and miR-122-5p on glucose metabolism of breast cancer cells by targeting HK2, LDHA, and PKM2, ECAR, ATP, and OCR were tested. MCF-7 and MDA-MB-231 cells were transfected with miR-216b-5p, miR-33a-5p and miR-122-5p combined with HK2, LDHA, and PKM2. It was found that miR-216b-5p, miR-33a-5p and miR-122$5 \mathrm{p}$ could reduce HK2, LDHA or PKM2 mediated ECAR increasement (Figure 7A-7C). ATP production decreased in the cells with HK2, LDHA or PKM2 overexpression in breast cancer cells with miR-216b-5p, miR-33a-5p and miR-122-5p overexpression (Figure 7D-7F). It was found
A

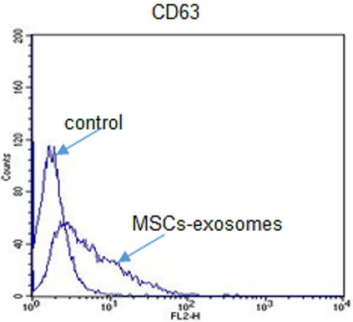

C

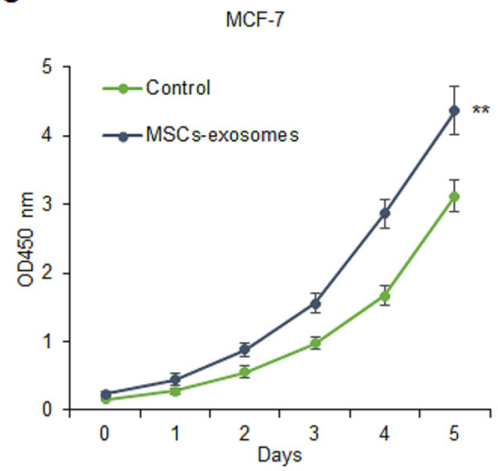

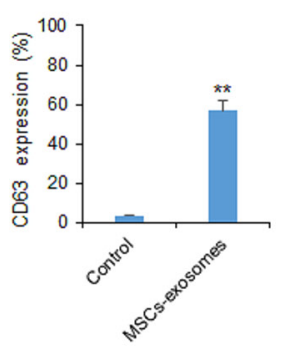

D
$\mathrm{B}$

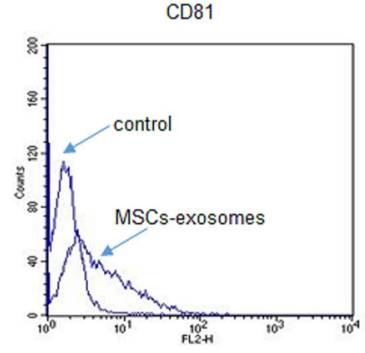

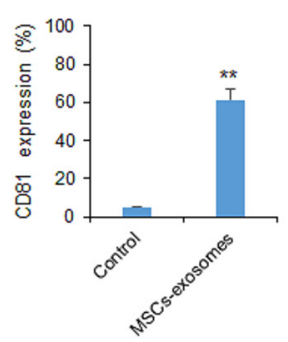

E
MDA-MB-468
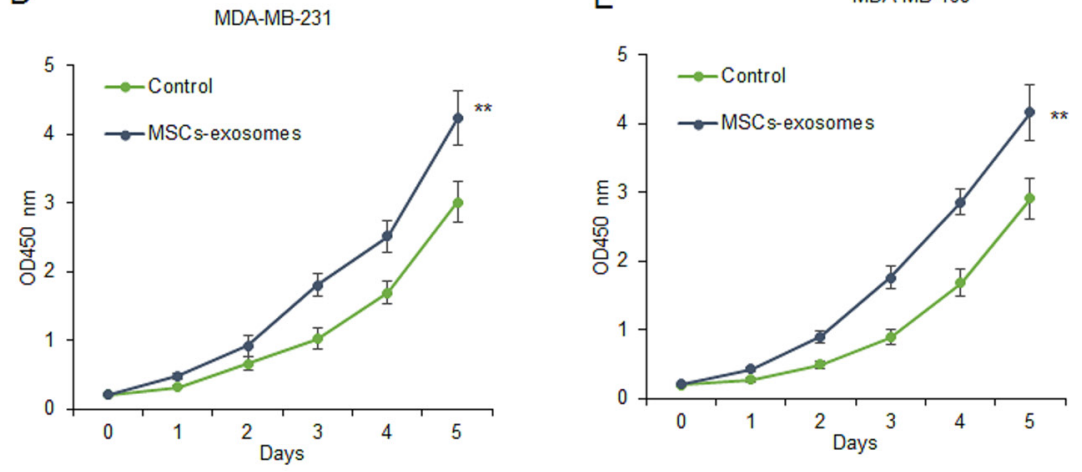

Figure 1: MSCs-derived exosomes promotes breast cancer cell proliferation. (A-B) The markers of exosomes including CD63 and CD81 were examined by Flow cytometry. (C-E) Breast cancer cell proliferation was assayed by MTT method. MCF-7, MDA-MB-468 and MDA-MB-231 cells were exposed to exosomes from MSCs for 1, 2, 3, 4 and 5 days and then measure cell survival rates. ${ }^{* *}$ p $<0.01$; " $\mathrm{p}<0.05$. 
A

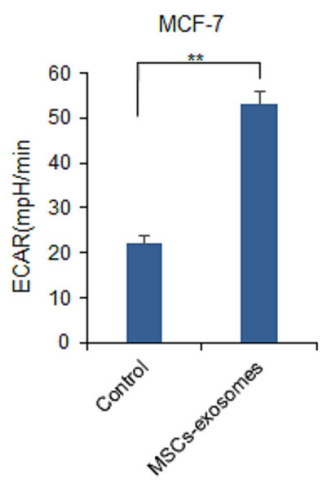

D

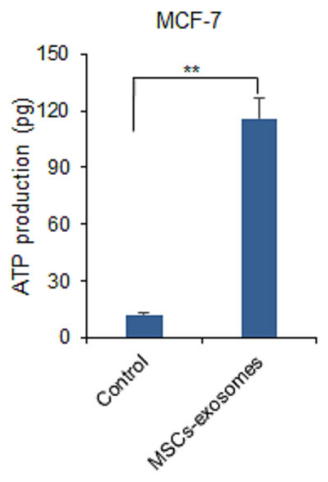

G

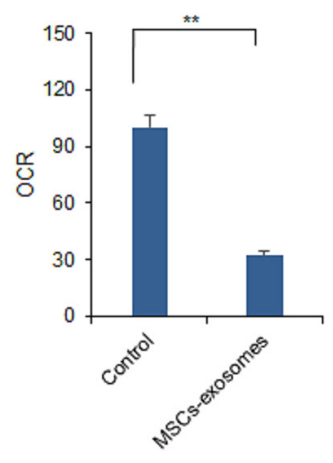

B

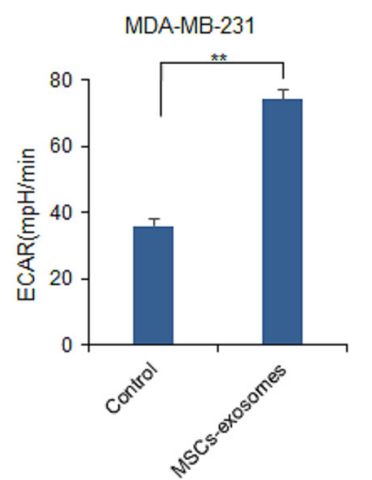

E

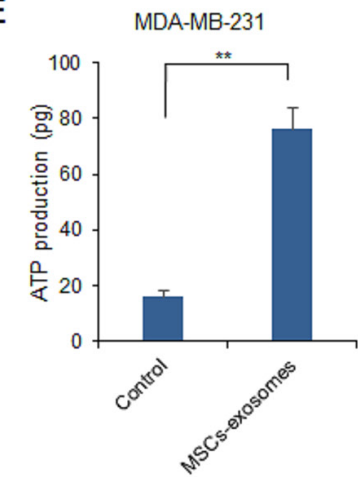

$\mathrm{H}$

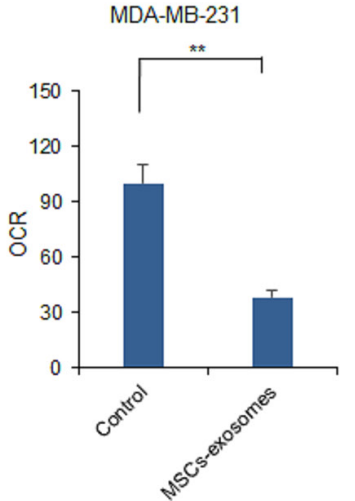

C

MDA-MB-468

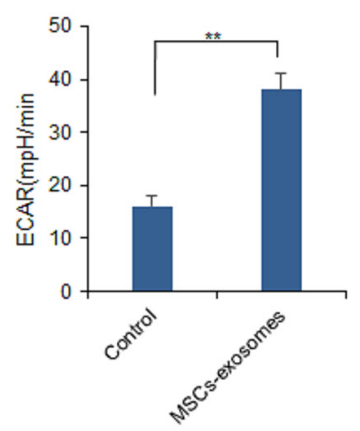

F
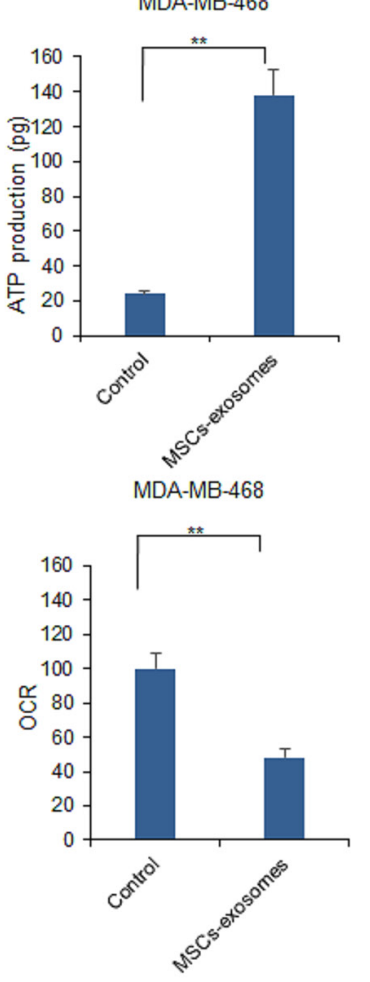

J

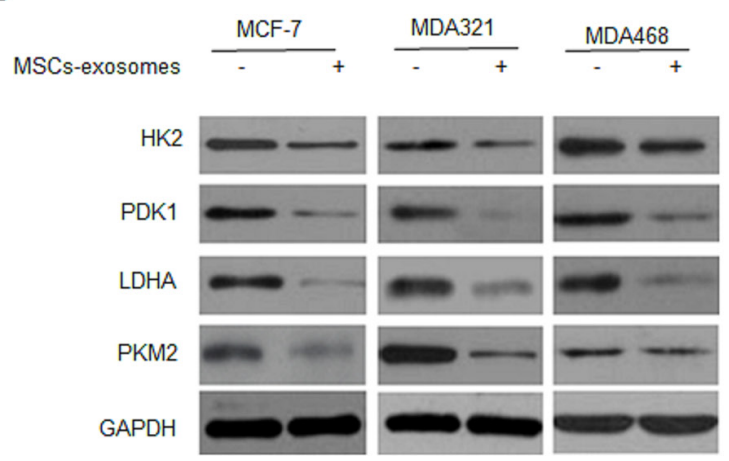

Figure 2: MSCs-derived exosomes promotes breast cancer cell glycolysis. (A-C) ECAR was measured in purified primary breast cancer cells following a 3-day culture in the presence or absence of MSCs-exosomes under basal conditions. (D-F) ATP production was measured in purified primary breast cancer cells following a 3-day culture in the presence or absence of MSCs-exosomes under basal conditions. (G-I) OCR was measured in purified primary breast cancer cells following a 3-day culture in the presence or absence of MSCsexosomes under basal conditions. (J) Changes in the relative gene expression of key glycolytic enzymes were determined by qPCR in breast cancer cells upon exosomes contact for 3 days as compared with cells cultured alone. 
that miR-216b-5p, miR-33a-5p and miR-122-5p could increase OCR of the breast cancer cells with HK2, LDHA or PKM2 overexpression (Figure 7G-7I).

\section{DISCUSSION}

MSCs is attractive in the field of tumor environment. MSCs in tumor microenvironment are from bone marrow or other tissues. MSCs integrate into the tumor stroma and function in a paracrine manner to promote breast cancer progression. The secreting paracrine molecules is a main functional way in tumor stroma $[11,12]$. Although there are studies indicate the important roles of MSCs in breast cancer, its role in glycolysis is still unclear and the molecular mechanisms mediating breast cancer cell glycolysis needs to be investigated. In this study, our purpose is to investigate the interaction between MSCs and breast cancer cells. Through isolating exosomes from the conditioned medium from MSCs, exosomes were identified according to the markers. Further studies focused on the role and molecular mechanism of exosomes on breast cancer cell glycolysis.
Exosomes may act as a source of metabolites and proliferating cancer. Firstly, the exosomes was isolated from the conditioned medium from exosomes of MSCs. Based on the identification of exosomes, breast cancer cell proliferation was assayed. We found that MSCs could promoted breast cellular survival ability. The results were similar to the reports [13]. The Warburg effect is a characteristic of cancer. After isolating exosomes from MSCs, we measured lactate acetate, ECAR, and ATP production of breast cancer cells with exosomes treatment. The data clearly demonstrate that exosomes from MSCs increased breast cancer cell glycolysis.

Exosomes are known to carry a complex cargo that includes proteins, lipids and miRNAs. The miRNA array indicated that there were many significant increased or decreased miRNAs from the MSCs derived exosomes. Our results from the array demonstrated that miR-216b$5 p$, miR-33a-5p and miR-122-5p levels were low in MSCs secrete exosomes. The target genes of miR-216b$5 p$, miR-33a-5p and miR-122-5p were searched using the tools online and we found that the most potential target genes of them were HK2, LDHA and PKM2 respectively.

A

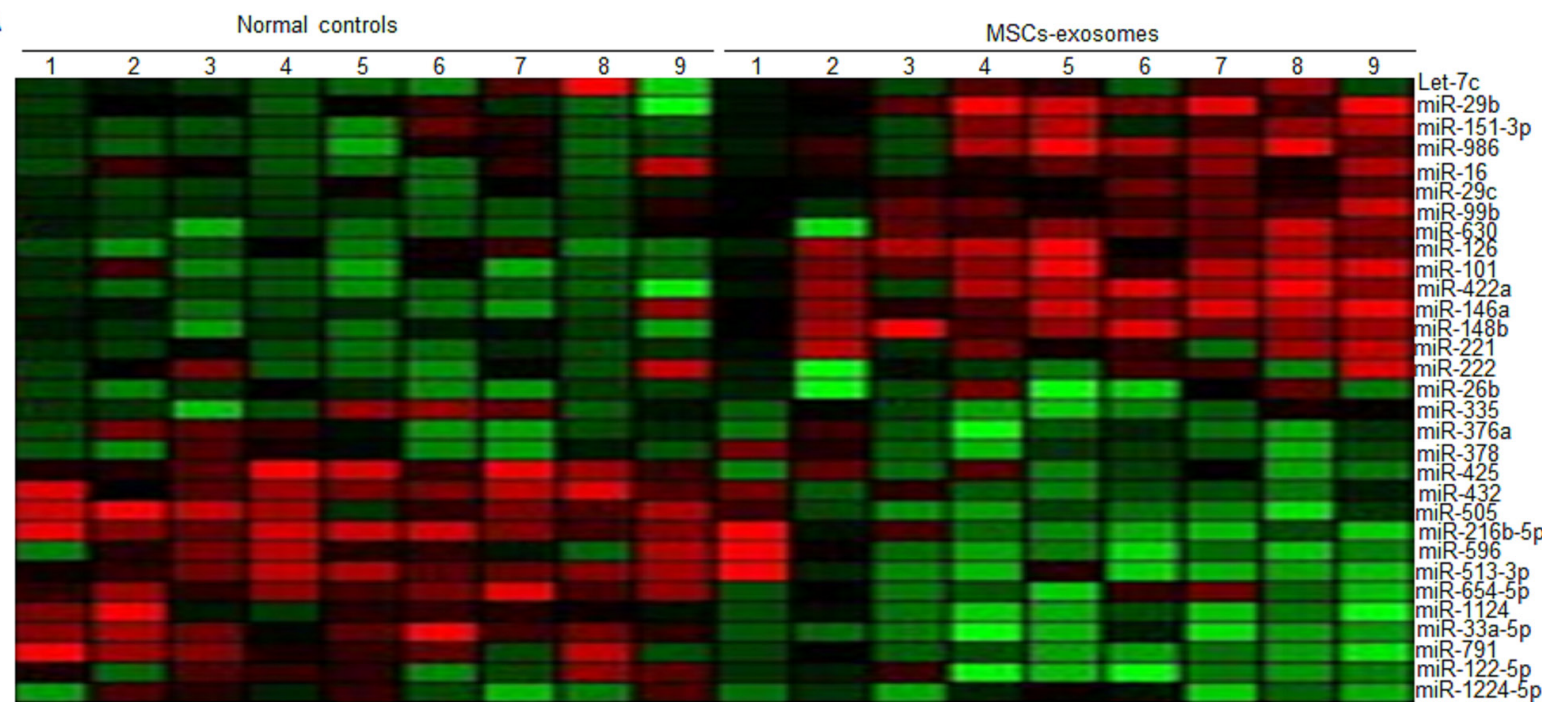

B

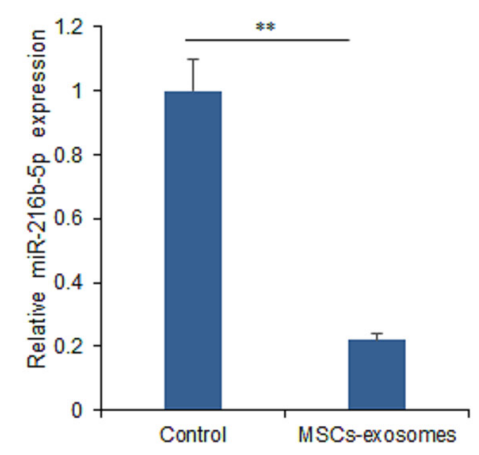

C

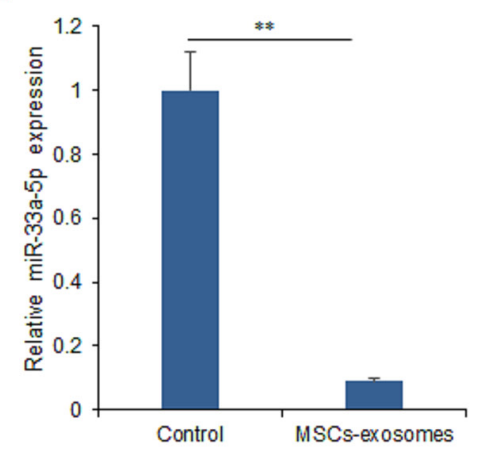

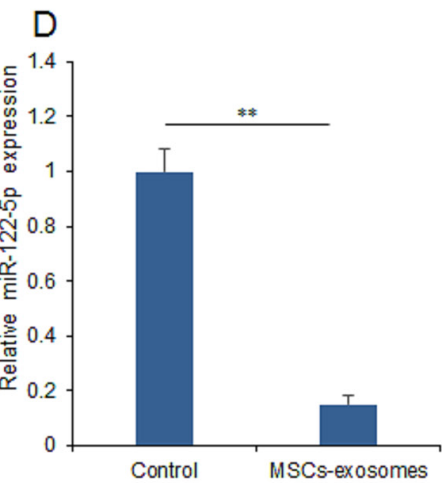

Figure 3: MSCs-derived exosomes carrying some significant miRNAs. (A) MiRNA expression map. 1-9 indicated the different samples from the breast cancer patients. (B-D) miR-216b-5p, miR-33a-5p and miR-122-5p expression were selected for real time RT-PCR verification in MSC-exosomes. 
HK2, LDHA and PKM2 were verified as the target genes of miR-216b-5p, miR-33a-5p and miR-122-5p using the method of luciferase assay. HK2 and LDHA mRNA and protein levels decreased in breast cancer cells with miR-
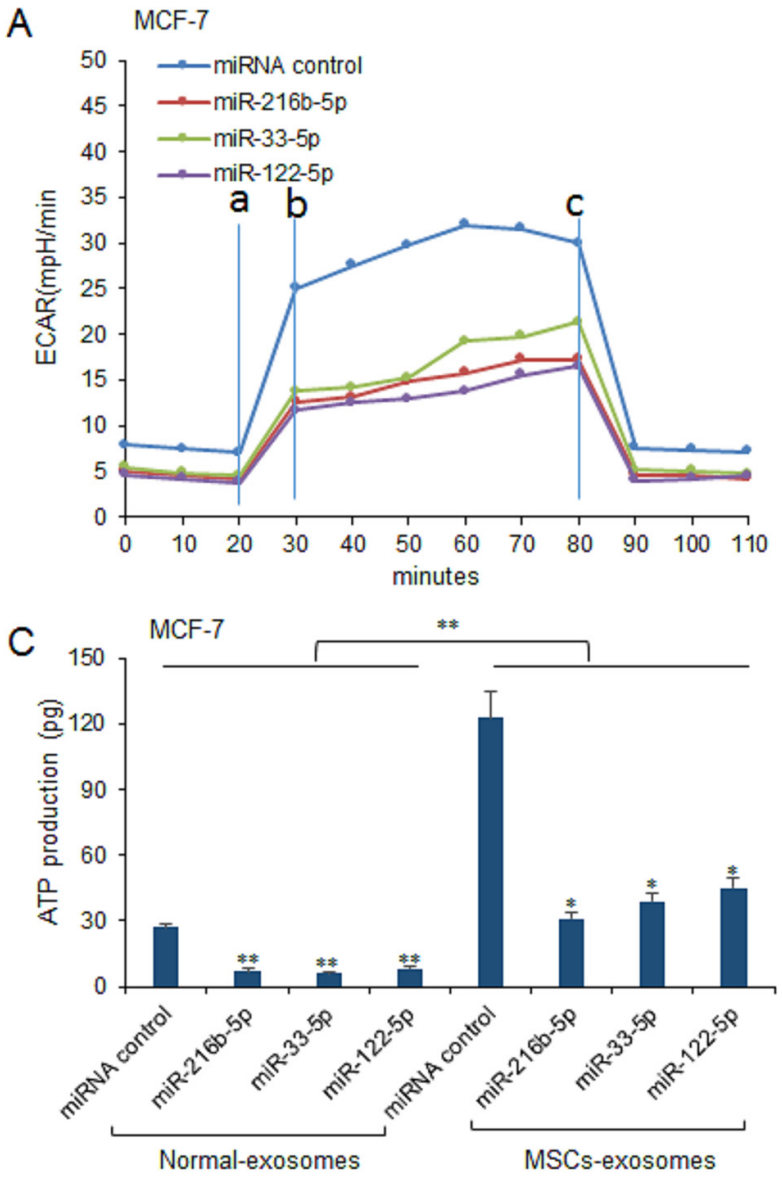

E MCF-7

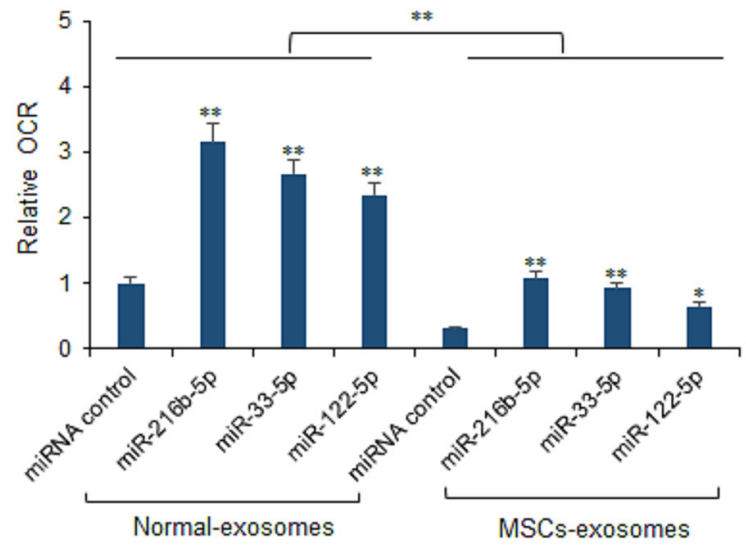

$216 b-5 p$ and miR-33a-5p mimics transfection. There was no significant difference for PKM2 mRNA in the breast cancer with miR-122-5p or the miRNA control transfection, but, PKM2 protein showed down-regulation
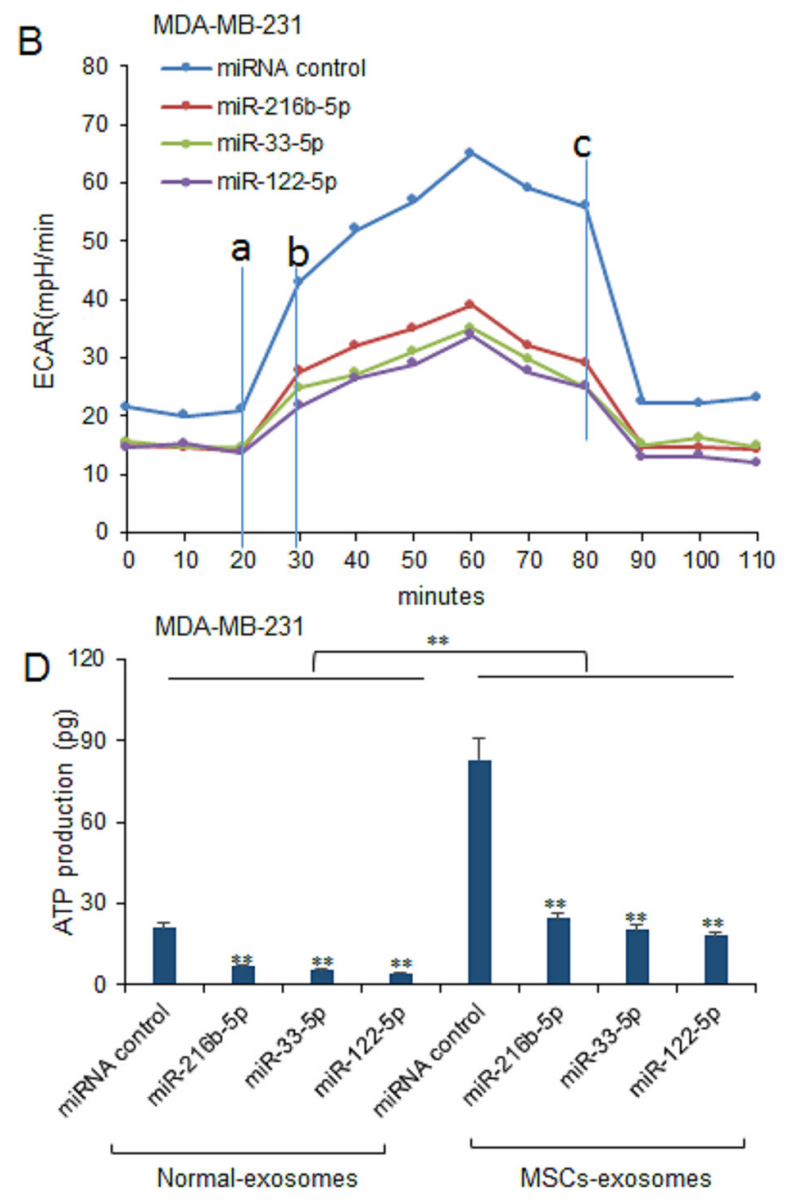

F $\quad$ MDA-MB-231

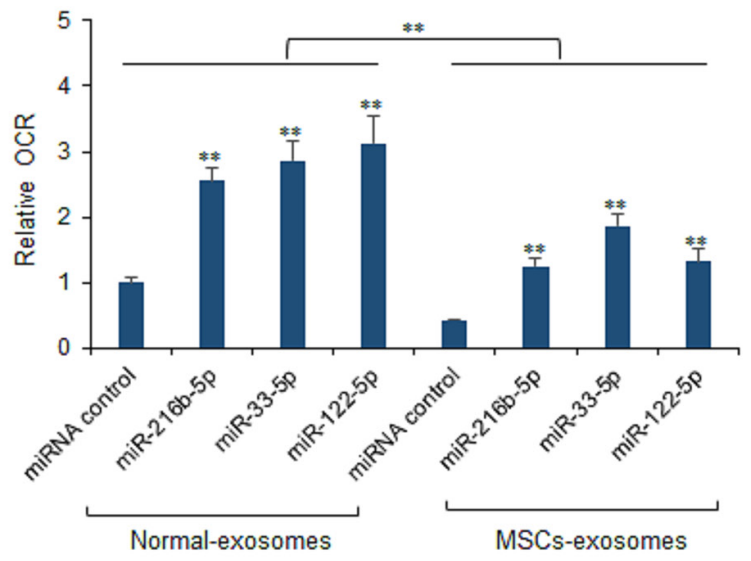

Figure 4: MiR-216b-5p, miR-33 and miR-122-5p contribute to exosomes-mediated glucose metabolism. (A-B) The ECAR was evaluated in MCF-7 and MDA-MB-231 cells transfected with miR-216b-5p, miR-33a-5p and miR-122-5p. a: glucose; b: Oligomycin; c: 2-DG. (C-D) ATP production was measured in MCF-7 and MDA-MB-231 cells transfected with miR-216b-5p, miR-33a-5p and miR122-5p under basal conditions. (E-F) OCR was measured in MCF-7 and MDA-MB-231 cells transfected with miR-216b-5p, miR-33a-5p and miR-122-5p under basal conditions. Figure 4 legend: is this fold-change of expression - define how normalized. 
A

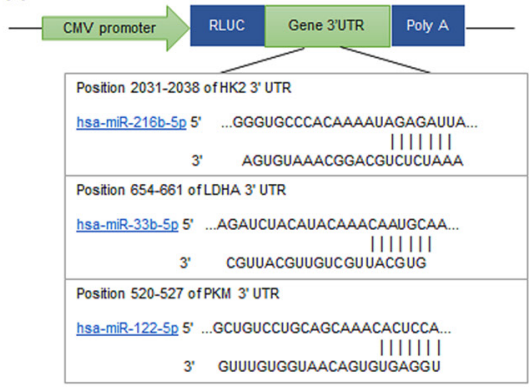

B

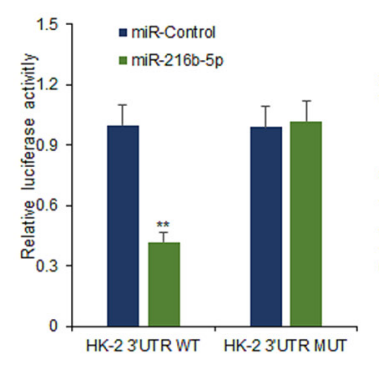

C

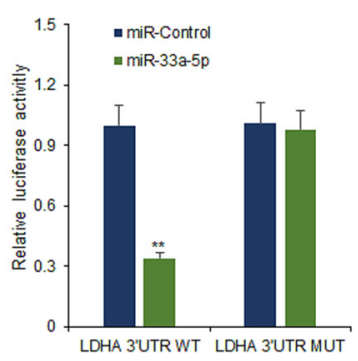

D

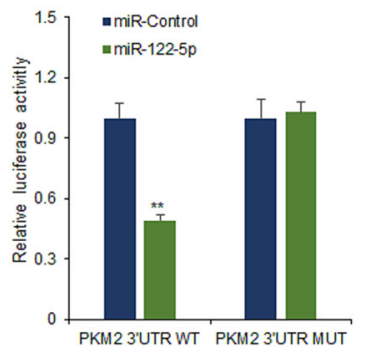

Figure 5: Target glucose associated genes of miR-216b-5p, miR-33a-5p and miR-122-5p in breast cancer cells. (A) Schematic representation of 3'-UTR of mRNA reporter with the miR-216b-5p, miR-33a-5p and miR-122-5p seed-binding sites. (B-D) Luciferase activity assay of HEK293T cells transfected with luciferase constructs containing WT-3'UTR and Mut-3'UTR of HK2, LDHA and PKM2.
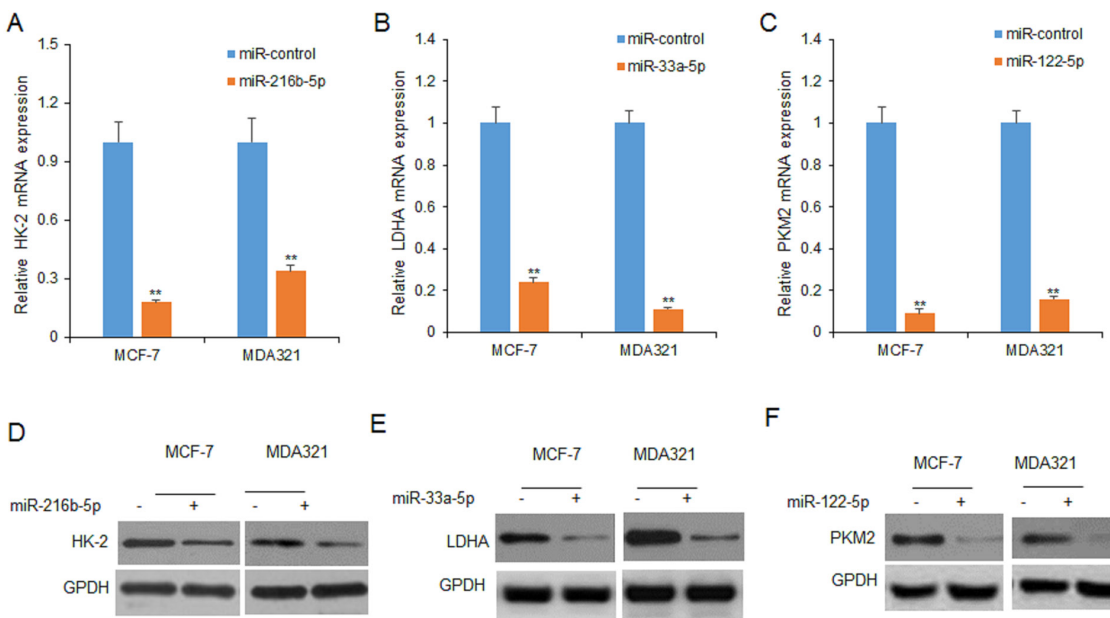

E

F
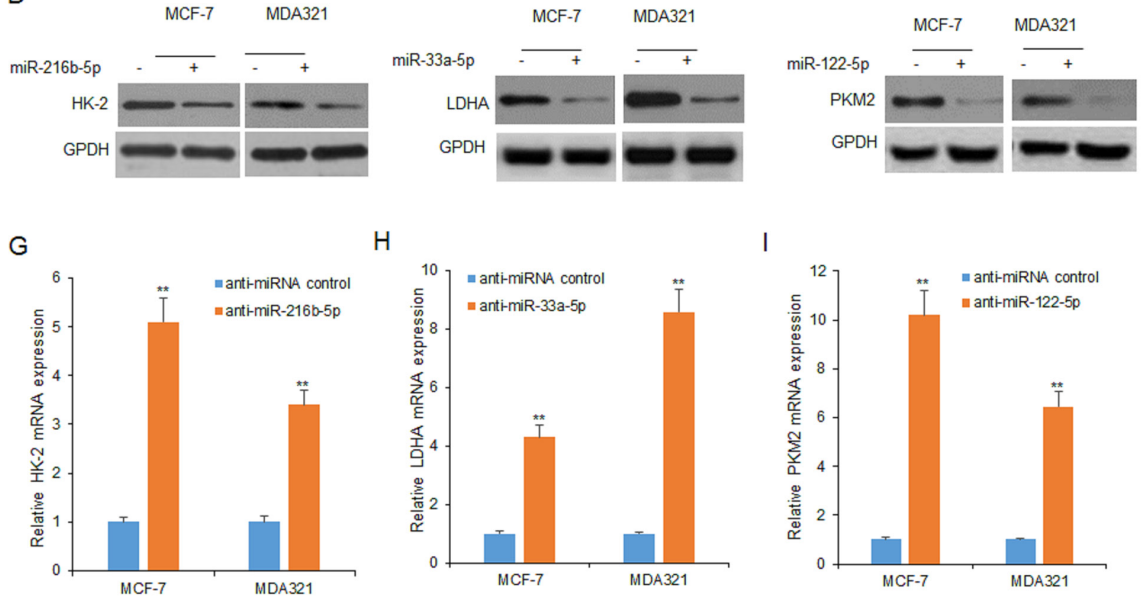

$\mathrm{H}$
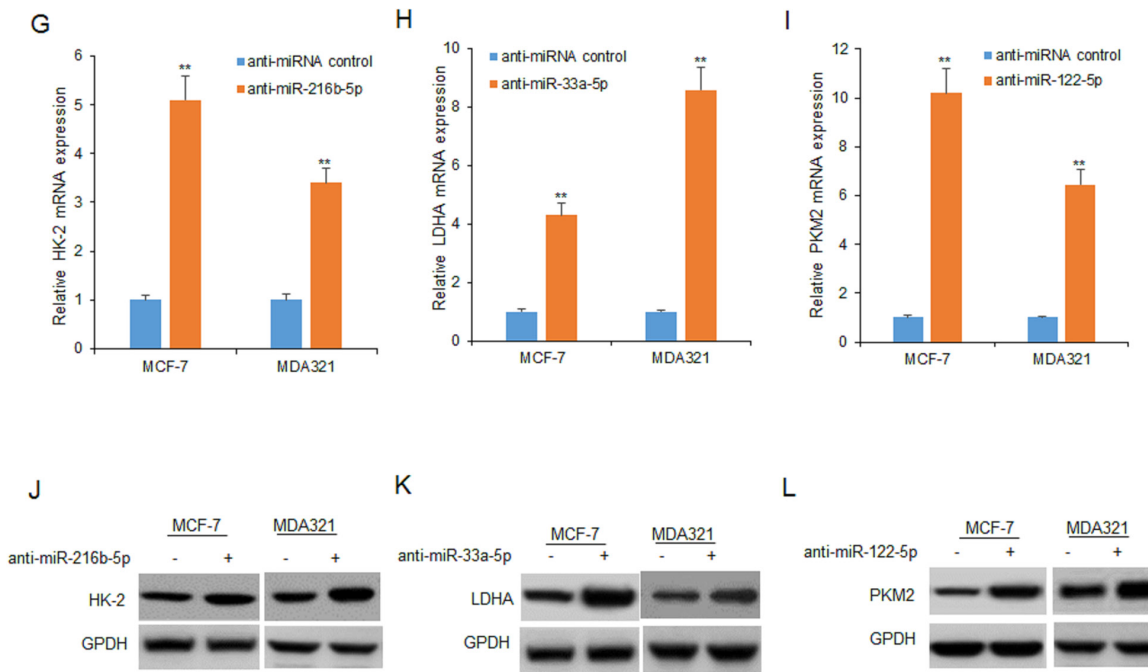

K

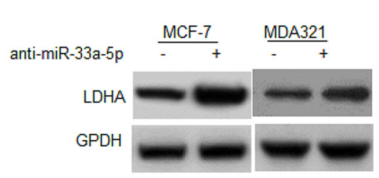

L

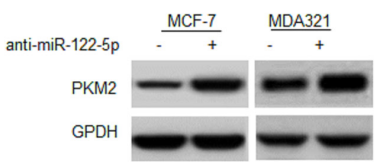

Figure 6: MiR-216b-5p, miR-33a-5p and miR-122-5p regulate HK2, LDHA and PKM2 expression in breast cancer cells. (A-C) HK2, LDHA and PKM2 mRNA were determined by qPCR in MCF-7 and MDA-MB-231 cells transfected with miR-216b-5p, miR-33a-5p and miR-122-5p mimics or their controls. (D-F) HK2, LDHA and PKM2 protein levels were determined by western blotting in MCF-7 and MDA-MB-231 cells transfected with miR-216b-5p, miR-33a-5p and miR-122-5p mimics or their controls. (G-I) HK2, LDHA and PKM2 mRNA were determined by qPCR in MCF-7 and MDA-MB-231 cells transfected with miR-216b-5p, miR-33a-5p and miR-122-5p inhibitors or their controls. (J-L) HK2, LDHA and PKM2 protein levels were determined by western blotting in MCF-7 and MDA-MB-231 cells transfected with miR-216b-5p, miR-33a-5p and miR-122-5p inhibitors or their controls. 
in the cells. HK2 was regulated by miR-143 [24], miR-155 [25], miR-199a-5p [26], miR-29b [27, 28-43], miR-140$5 \mathrm{p}$ in various cancers like bladder cancer, liver cancer and prostate cancer. LDHA was regulated by miR-383, miR34a, miR-122, miR-30a-5p and other miRNAs in cancer. LDHA plays roles in cell growth, invasion and aerobic glycolysis. PKM2 was regulated by miR-let-7a, miR124, miR-122, miR-124, miR-326, miR-290 and miR371 in cervical cancer, pancreatic cancer, hepatocellular carcinoma, colorectal cancer, glioma and esophageal cancer. PKM2 involves in cancer cell proliferation, metastasis, apoptosis and metabolism.

MiRNAs play great important roles in breast cancer glycolysis [14-19]. In this study, we found that miR-216b$5 \mathrm{p}$, miR-33a-5p and miR-122-5p inhibited glycolysis via targeting HK2, LDHA and PKM2. MiR-216b-5p is down-regulated in liver cancer cells [20], however, its role in breast cancer is not known. Our study for the first time verified that miR-216b-5p functioned as a tumor suppressing miRNA in breast cancer. MiR-33a-5p suppresses osteoscarcoma and hepatocellular carcinoma cell growth [21-22]. MiR-122-5p is down-regulated in breast cancer cell [23].

In a conclusion, our findings indicated that exosomes promote breast cancer cell glycolysis via the lack of miR-216b-5p, miR-33a-5p and miR-122-5p expression in exosomes derived from MSCs conditioned medium. MiR-216b-5p, miR-33a-5p and miR-122-5p functioned as glycolytic molecule by targeting $\mathrm{HK} 2$, LDHA and PKM2 expression respectively. The overall importance of this work is that the lack of miRNAs like miR-216b-5p, miR-33a-5p and miR-122-5p in MSCs
A

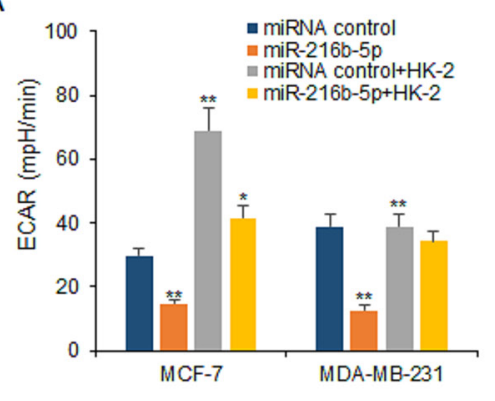

D

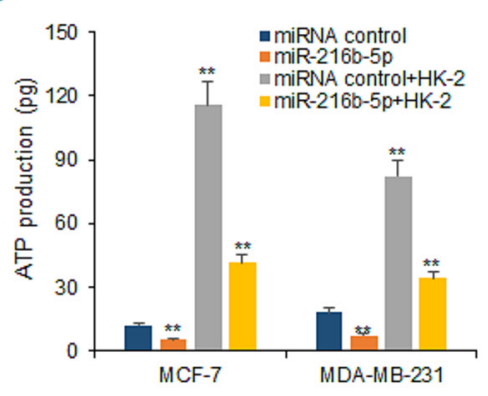

G

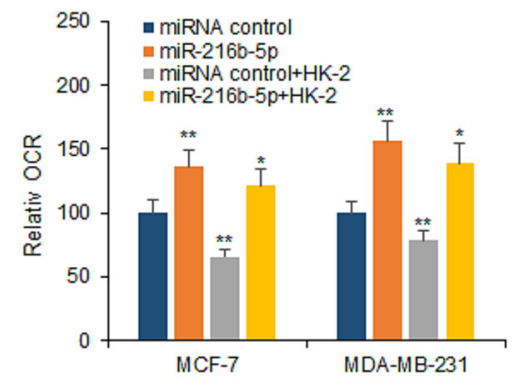

B

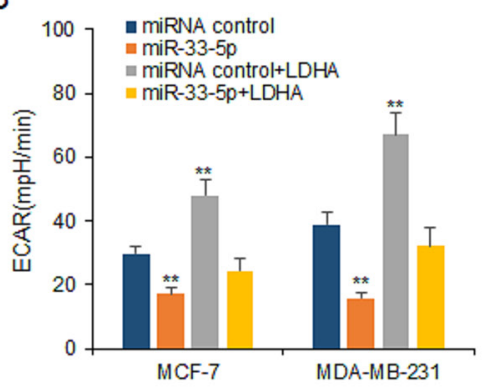

$\mathrm{E}$

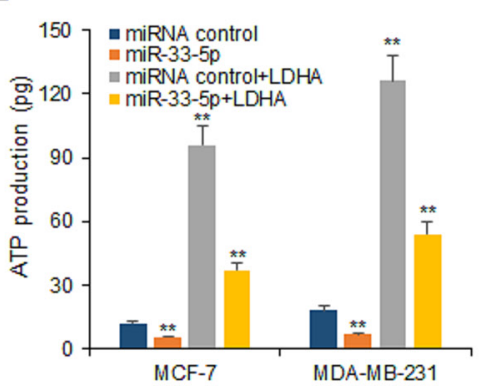

$\mathrm{H}$

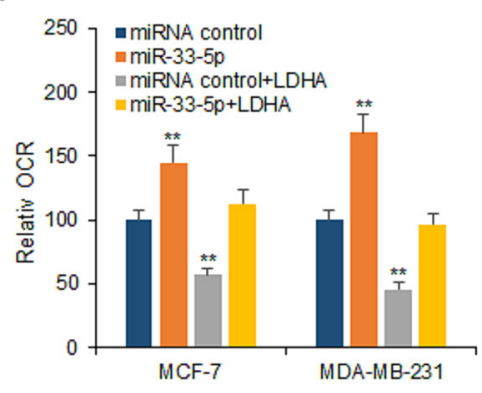

C

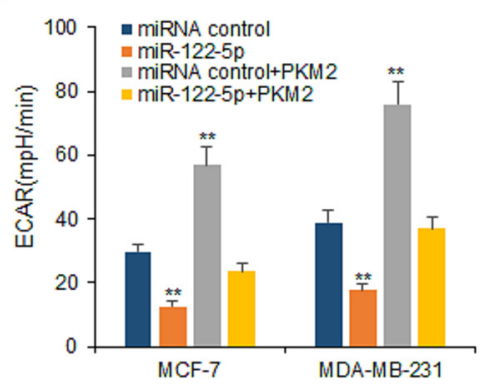

$\mathrm{F}$

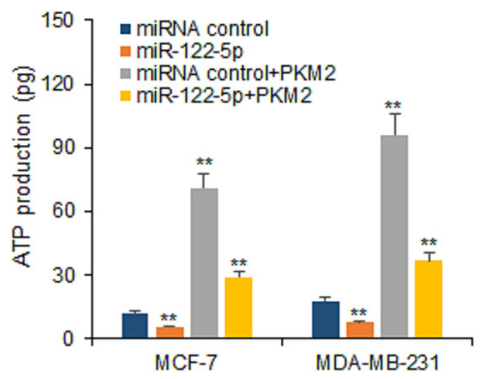

।

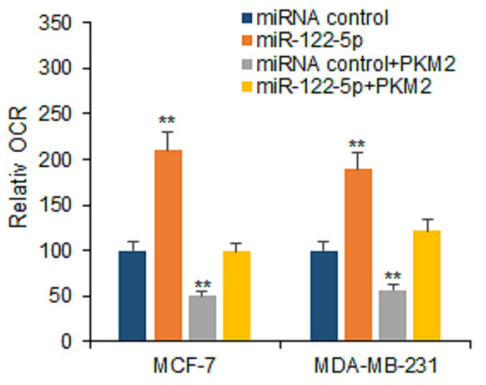

Figure 7: MiR-216b-5p, miR-33 and miR-122-5p contribute to exosomes-mediated glucose metabolism by targeting HK2, LDHA, and PKM2 in breast cancer cells. (A-C) The ECAR was evaluated in MCF-7 and MDA-MB-231 cells transfected with miR-216b-5p, miR-33a-5p and miR-122-5p combined with HK2, LDHA, and PKM2. (D-F) ATP production was measured in MCF-7 and MDA-MB-231 cells transfected with miR-216b-5p, miR-33a-5p and miR-122-5p under basal conditions combined with HK2, LDHA, and PKM2. (G-I) OCR was measured in MCF-7 and MDA-MB-231 cells transfected with miR-216b-5p, miR-33a-5p and miR-122-5p under basal conditions combined with HK2, LDHA, and PKM2. 
secreted exosomes. There needs further research on the mechanisms of MSCs derived exosomes involving in breast cancer behavior.

\section{MATERIALS AND METHODS}

\section{Cell culture}

MCF-7, MDA-MB-231 and MDA-MB-468 cells were purchased from and cultured as instructed by ATCC. Cells were maintained in DMEM with $10 \%$ fetal bovine serum, 2\% glutamine, and 1\% antibiotics (Invitrogen) under a humidified atmosphere of $5 \% \mathrm{CO}_{2}$ at $37{ }^{\circ} \mathrm{C}$. Medium was changed every 2-3 days. For experiments, cancer cells reached to $70-80 \%$ confluency.

\section{Conditioned medium preparation for exosomes extraction}

MSCs were plated and cultured in DMEM/F12 media with 10\% FBS for 24 hours, and then washed for three times with PBS and finally cultured in $3 \mathrm{ml}$ serum free DMEM/F12 media for 2 hours. The conditioned medium was collected and filtered through a $0.22-\mu \mathrm{m}$ filter (Merck Millipore, Massachusetts, USA) to remove cellular debris for extracting exosomes.

\section{Isolation and characterization of exosomes released by MSCs}

Exosomes were isolated from cultured MSCs by using Total Exosome Isolation Kit (Invitrogen, California, USA). Cells were grown in exosome-depleted media, and exosomes were isolated from the conditioned media. After incubated overnight at $4^{\circ} \mathrm{C}$, the mixture was centrifuged at $12,000 \times \mathrm{g}$ for $70 \mathrm{~min}$ at $4^{\circ} \mathrm{C}$ and all supernatant was removed by aspiration. Exosome pellets were suspended with a convenient volume of DMEM/F12 medium. For exosome assays, an equal volume of exosomes was added to cells for $24-48 \mathrm{~h}$. For exosome depletion, the conditioned medium was ultra-centrifuged overnight by serial centrifugation.

\section{MiRNA extraction and array assay}

MiRNAs were extracted from frozen pellets using mirVanaTM miRNA isolation kit (Life Technologies) according to manufacturer's instructions. Concentration was measured using Nanodrop 2000 (Thermo Scientific). The miRNA controls were from the normal MSCs. miRNA profiling was performed across a two-card set of TaqMan ${ }^{\circledR}$ MicroRNA Arrays (arrays A and B) (Life Technologies) for a total of 667 unique assays specific to human miRNAs (Sanger miRBase v10). Raw data (see Supplementary Table 1) were analyzed using both GenEX and RealTime StatMiner ${ }^{\circledR}$ software. Sample preparation was done according to the manufacturer's Protocol for Creating Custom RT and Preamplification Pools using Taqman ${ }^{\circledR}$ MicroRNA Assays (Life Technologies).

\section{Cell transfection}

Breast cancer cells were seeded in the cell plates and transfected with miRNAs (100nM) or the HK2, PKM2, or LDHA for 24-48h and then the cellular functions including cell proliferation, metastasis and glycolysis were arrayed. For luciferase assay, the concentration of the plasmids of HK2, LDHA and PKM2 3'UTR used in transfection were 100uM.

\section{Cell proliferation}

Breast cancer cells were seeded in 96-well plates and transfected with the indicated miRNAs or treated with exosomes and cultured in the normal condition. Cell survival ability was tested by MTT (Sigma) assay.

\section{Dual luciferase reporter assay}

Cells were seeded in 24-well plates and transfected the HK2, LDHA and PKM2 3'UTR luciferase reporter, wild type or mutant reporter constructs and Renilla plasmid by using lipofectamine 2000 (Invitrogen). The primers for HK2, LDHA and PKM2 3'UTR amplication were provided in Supplementary Table 2. HK2, LDHA and PKM2 3'UTR activity was examined using DualLuciferase Reporter Assay System (Promega) according to the manufacturer's instruction.

\section{RNA extraction and real-time PCR analysis}

Breast cancer cells were transfected with miR$216 b-5 p$, miR-33a-5p or miR-122-5p or the controls for $48 \mathrm{~h}$ and then total RNA was isolated for real time RT-PCR analysis. The expression level of miRNAs was defined based on the threshold cycle $(\mathrm{Ct})$, and relative expression levels were calculated using the $2^{-\Delta \Delta C t}$ method, using the expression level of the U6 snRNA as a reference gene.

\section{Western blotting}

Cultured cells were harvested and lysed with RIPA buffer containing the protease inhibitors on ice for $30 \mathrm{mins}$. Protein was separated by SDS-PAGE and transferred onto nitrocellulose membrane using and probed with primary antibodies including HK2, LDHA, PKM2 or GAPDH and then horseradish peroxidase-labeled secondary antibodies. The protein band signals were visualized using an ECL. All the primary and secondary antibodies were ordered from cell signaling technology (CST). 


\section{Statistical analysis}

All analysis were performed using the SPSS 15.0 (SPSS, Chicago, IL, USA) or Excel. Every experiment was completed independently at least three times. A $\mathrm{p}$ value $<0.05$ was considered significant.

\section{ACKNOWLEDGMENTS}

This study was supported by the mutual fund of Science\&Technology Department of Sichuan province (2015LZCYD-S01(12/15), The project of education department of Sichuan province (15TD0020), The project of Science\&Technology Department of Sichuan province (2013JY0076), The project of Science\&Technology Department of Luzhou City (2013LZLY-J12) and The major project of education department of Sichuan province (16ZA0189).

\section{CONFLICTS OF INTEREST}

The authors have no conflicts of interest.

\section{REFERENCES}

1. Sage EK, Thakrar RM, Janes SM. Genetically modified mesenchymal stromal cells in cancer therapy. Cytotherapy. 2016; 18:1435-1445.

2. Poggi A, Giuliani M. Mesenchymal stromal cells can regulate the immune response in the tumor microenvironment. Vaccines (Basel). 2016.

3. Shi S, Zhang Q, Xia Y, You B, Shan Y, Bao L, Li L, You Y, $\mathrm{Gu} \mathrm{Z}$. Mesenchymal stem cell-derived exosomes facilitate nasopharyngeal carcinoma progression. Am J Cancer Res. 2016; 6:459-72.

4. Dotto GP. Multifocal epithelial tumors and field cancerization: stroma as a primary determinant. J Clin Invest. 2014; 124:1446-53.

5. Wang Z, Chen JQ, Liu JL, Tian L. Exosomes in tumor microenvironment: novel transporters and biomarkers. J Transl Med. 2016; 14:297.

6. Santos JC, Ribeiro ML, Sarian LO, Ortega MM, Derchain SF. Exosomes-mediate microRNAs transfer in breast cancer chemoresistance regulation. Am J Cancer Res. 2016; 6:2129-2139.

7. Roma-Rodrigues C, Raposo LR, Cabral R, Paradinha F, Baptista PV, Fernandes AR. Tumor microenvironment modulation via gold nanoparticles targeting malicious exosomes: implications for cancer diagnostics and therapy. Int J Mol Sci. 2017; 18.

8. Wang J, Faict S, Maes K, De Bruyne E, Van Valckenborgh E, Schots R, Vanderkerken K, Menu E. Extracellular vesicle cross-talk in the bone marrow microenvironment: implications in multiple myeloma. Oncotarget. 2016; 7:38927-38945. https://doi.org/10.18632/oncotarget.7792.

9. Shao Y, Shen Y, Chen T, Xu F, Chen X, Zheng S. The functions and clinical applications of tumor-derived exosomes. Oncotarget. 2016; 7:60736-60751. https://doi. org/10.18632/oncotarget.11177.

10. Frediani JN, Fabbri M. Essential role of miRNAs in orchestrating the biology of the tumor microenvironment. Mol Cancer. 2016; 15:42.

11. Karnoub AE, Dash AB, Vo AP, Sullivan A, Brooks MW, Bell GW, Richardson AL, Polyak K, Tubo R, Weinberg RA. Mesenchymal stem cells within tumour stroma promote breast cancer metastasis. Nature. 2007; 449:557-563.

12. Luga V, Wrana JL. Tumor-stroma interaction: revealing fibroblast-secreted exosomes as potent regulators of Wntplanar cell polarity signaling in cancer metastasis. Cancer Res. 2013; 73:6843-7.

13. Ge X, Lyu P, Cao Z, Li J, Guo G, Xia W, Gu Y. Overexpression of miR-206 suppresses glycolysis, proliferation and migration in breast cancer cells via PFKFB3 targeting. Biochem Biophys Res Commun. 2015; 463:1115-21.

14. Serguienko A, Grad I, Wennerstrøm AB, Meza-Zepeda LA, Thiede B, Stratford EW, Myklebost O, Munthe E. Metabolic reprogramming of metastatic breast cancer and melanoma by let-7a microRNA. Oncotarget. 2015; 6:245165. https://doi.org/10.18632/oncotarget.3235.

15. Fong MY, Zhou W, Liu L, Alontaga AY, Chandra M, Ashby J, Chow A, O'Connor ST, Li S, Chin AR, Somlo G, Palomares M, Li Z, et al. Breast-cancer-secreted miR-122 reprograms glucose metabolism in premetastatic niche to promote metastasis. Nat Cell Biol. 2015; 17:183-94.

16. Verghese ET, Drury R, Green CA, Holliday DL, Lu X, Nash C, Speirs V, Thorne JL, Thygesen HH, Zougman A, Hull MA, Hanby AM, Hughes TA. MiR-26b is down-regulated in carcinoma-associated fibroblasts from ER-positive breast cancers leading to enhanced cell migration and invasion. $\mathrm{J}$ Pathol. 2013; 231:388-99.

17. Xiao X, Huang X, Ye F, Chen B, Song C, Wen J, Zhang J, Zheng G, Tang H, Xie X. The miR-34a-LDHA axis regulates glucose metabolism and tumor growth in breast cancer. Sci Rep. 2016; 6:21735.

18. Ahmad A, Aboukameel A, Kong D, Wang Z, Sethi S, Chen W, Sarkar FH, Raz A. Phosphoglucose isomerase/autocrine motility factor mediates epithelial-mesenchymal transition regulated by miR-200 in breast cancer cells. Cancer Res. 2011; 71:3400-9.

19. Dluzen DF, Sutliff AK, Chen G, Watson CJ, Ishmael FT, Lazarus P. Regulation of UGT2B expression and activity by miR-216b-5p in liver cancer cell lines. J Pharmacol Exp Ther. 2016; 359:182-93.

20. Fang Y, Feng Y, Wu T, Srinivas S, Yang W, Fan J, Yang C, Wang S. Aflatoxin B1 negatively regulates Wnt/ $\beta$-catenin 
signaling pathway through activating miR-33a. PLoS One. 2013; 8:e73004.

21. Zhang J, Wang D, Xiong J, Chen L, Huang J. MicroRNA$33 a-5 p$ suppresses growth of osteosarcoma cells and is downregulated in human osteosarcoma. Oncol Lett. 2015; 10:2135-2141.

22. Ergün S, Ulasli M, Igci YZ, Igci M, Kırkbes S, Borazan E, Balik A, Yumrutaş Ö, Camci C, Cakmak EA, Arslan A, Oztuzcu S. The association of the expression of miR-122-5p and its target ADAM10 with human breast cancer. Mol Biol Rep. 2015; 42:497-505.

23. Jiang S, Zhang LF, Zhang HW, Hu S, Lu MH, Liang S, Li B, Li Y, Li D, Wang ED, Liu MF. A novel miR-155/miR143 cascade controls glycolysis by regulating hexokinase 2 in breast cancer cells. EMBO J. 2012; 31:1985-98.

24. Li Z, Li X, Wu S, Xue M, Chen W. Long non-coding RNA UCA1 promotes glycolysis by upregulating hexokinase 2 through the mTOR-STAT3/microRNA143 pathway. Cancer Sci. 2014; 105:951-5.

25. Guo W, Qiu Z, Wang Z, Wang Q, Tan N, Chen T, Chen Z, Huang S, Gu J, Li J, Yao M, Zhao Y, He X. MiR-199a-5p is negatively associated with malignancies and regulates glycolysis and lactate production by targeting hexokinase 2 in liver cancer. Hepatology. 2015; 62:1132-44.

26. Wang Y, Lu X, He J, Zhao W. Influence of erythropoietin on microvesicles derived from mesenchymal stem cells protecting renal function of chronic kidney disease. Stem Cell Res Ther. 2015; 6:100.

27. Zhou P, Chen WG, Li XW. MicroRNA-143 acts as a tumor suppressor by targeting hexokinase 2 in human prostate cancer. Am J Cancer Res. 2015; 5:2056-63.

28. Zhang LF, Lou JT, Lu MH, Gao C, Zhao S, Li B, Liang S, Li Y, Li D, Liu MF. Suppression of miR-199a maturation by HuR is crucial for hypoxia-induced glycolytic switch in hepatocellular carcinoma. EMBO J. 2015; 34:2671-85.

29. Teng Y, Zhang Y, Qu K, Yang X, Fu J, Chen W, Li X. MicroRNA-29B (mir-29b) regulates the Warburg effect in ovarian cancer by targeting AKT2 and AKT3. Oncotarget. 2015; 6:40799-814. https://doi.org/10.18632/ oncotarget.5695.

30. Liao W, Fu Z, Zou Y, Wen D, Ma H, Zhou F, Chen Y, Zhang M, Zhang W. MicroRNA-140-5p attenuated oxidative stress in Cisplatin induced Acute Kidney Injury by activating Nrf2/ARE pathway through a Keap1-independent mechanism. Exp Cell Res. 2017 Sep 16.

31. Han RL, Wang FP, Zhang PA, Zhou XY, Li Y. miR-383 inhibits ovarian cancer cell proliferation, invasion and aerobic glycolysis by targeting LDHA. Neoplasma. 2017; 64:244-252. 28043152.

32. Zhang R, Su J, Xue SL, Yang H, Ju LL, Ji Y, Wu KH, Zhang YW, Zhang YX, Hu JF, Yu MM. HPV E6/p53 mediated down-regulation of miR-34a inhibits Warburg effect through targeting LDHA in cervical cancer. Am J Cancer Res. 2016; 6:312-20.
33. Song K, Kwon H, Han C, Zhang J, Dash S, Lim K, $\mathrm{Wu}$ T. Active glycolytic metabolism in CD133(+) hepatocellular cancer stem cells: regulation by MIR-122. Oncotarget. 2015; 6:40822-35. https://doi.org/10.18632/ oncotarget.5812.

34. Li L, Kang L, Zhao W, Feng Y, Liu W, Wang T, Mai H, Huang J, Chen S, Liang Y, Han J, Xu X, Ye Q. miR-30a-5p suppresses breast tumor growth and metastasis through inhibition of LDHA-mediated Warburg effect. Cancer Lett. 2017; 400:89-98.

35. Xiao X, Huang X, Ye F, Chen B, Song C, Wen J, Zhang Z, Zheng G, Tang H, Xie X. The miR-34a-LDHA axis regulates glucose metabolism and tumor growth in breast cancer. Sci Rep. 2016; 6:21735.

36. Guo M, Zhao X, Yuan X, Jiang J, Li P. MiR-let-7a inhibits cell proliferation, migration, and invasion by downregulating PKM2 in cervical cancer. Oncotarget. 2017; 8:28226-28236. https://doi.org/10.18632/oncotarget.15999.

37. Li C, Zhao Z, Zhou Z, Liu R. Linc-ROR confers gemcitabine resistance to pancreatic cancer cells via inducing autophagy and modulating the miR-124/ PTBP1/PKM2 axis. Cancer Chemother Pharmacol. 2016; 78:1199-1207.

38. Xu Q, Zhang M, Tu J, Pang L, Cai W, Liu X. MicroRNA-122 affects cell aggressiveness and apoptosis by targeting PKM2 in human hepatocellular carcinoma. Oncol Rep. 2015; 34:2054-64.

39. Taniguchi K, Sugito N, Kumazaki M, Shinohara H, Yamada N, Nakagawa Y, Ito Y, Otsuki Y, Uno B, Uchiyama K, Akao Y. MicroRNA-124 inhibits cancer cell growth through PTB1/PKM1/PKM2 feedback cascade in colorectal cancer. Cancer Lett. 2015; 363:17-27.

40. Kefas B, Comeau L, Erdle N, Montgomery E, Amos S, Purow B. Pyruvate kinase M2 is a target of the tumorsuppressive microRNA-326 and regulates the survival of glioma cells. Neuro Oncol. 2010; 12:1102-12.

41. Liu AM, Xu Z, Shek FH, Wong KF, Lee NP, Poon RT, Chen J, Luk JM. miR-122 targets pyruvate kinase M2 and affects metabolism of hepatocellular carcinoma. PLoS One. 2014; 9:e86872.

42. Cao Y, Guo WT, Tian S, He X, Wang XW, Liu X, Gu KL, Ma X, Huang D, Hu L, Cai Y, Zhang H, Wang Y, Gao P. miR-290/371-Mbd2-Myc circuit regulates glycolytic metabolism to promote pluripotency. EMBO J. 2015; 34:609-23.

43. Zhang HS, Zhang FJ, Li H, Liu Y, Du GY, Huang YH. Tanshinone IIA inhibits human esophageal cancer cell growth through miR-122-mediated PKM2 down-regulation. Arch Biochem Biophys. 2016; 598:50-6. 